\title{
Efecto de la calidad espectral sobre el desarrollo vegetal de Salvia splendens variedad Vista Red and White
}

\section{Effect of spectral quality on plant development of Salvia splendens variety Vista Red and White}

Federico José Gómez-Coto'

Fecha de recepción: 3 de febrero del 2014 Fecha de aprobación: I I de agosto del 2014

Gómez, F. Efecto de la calidad espectral sobre el desarrollo vegetal de Salvia splendens variedad

Vista Red and White. Tecnología en Marcha. Edición Especial Movilidad Estudiantil 20I4. Pág

49-54. 


\section{Palabras clave}

Calidad espectral; fuente lumínica; longitud de onda; Salvia; Scarlet Sage.

\section{Resumen}

Tres diferentes calidades de luz fueron analizadas sobre una especie ornamental de importancia económica y comercial: Salvia splendens ó Scarlet Sage (variedad Vista Red \& White de PanAmerican Seeds(C), mediante la utilización de tres distintas fuentes de luz artificial (azul tipo LED, púrpura tipo LED y fluorescente) para modificar el ambiente de crecimiento de cada una de ellas. La investigación abarcó el análisis de la duración de días a floración, la altura, el diámetro, número de brotes y valores SPAD. Estudios existentes acerca del efecto de la calidad espectral sobre el desarrollo vegetal demuestran que hay alteraciones significativas en variables fotomorfogénicas y de crecimiento vegetal. Por ejemplo en el peso seco de la planta, altura, longitud entrenudos, número de brotes, duración del periodo de floración, número de estomas por planta y cantidad de almidones y azúcares reductores. Sin embargo, en el estudio realizado se encontró que la calidad espectral proporcionada con luz suplementaria sólo tiene efecto en los días a floración, comparado con el tratamiento testigo, puesto que reduce en una semana la floración, pero, no hay diferencia entre los tres diferentes tratamientos con luz suplementaria. La demás variables analizadas (número de brotes, altura, diámetro promedio y valores SPAD) no tienen un efecto significativo por la calidad espectral utilizada ( $p>0.05)$. El estudio fue realizado en el invernadero académico del Tecnológico de Monterrey, ubicado en Monterrey, Nuevo León, México; durante el periodo de Agosto-Diciembre, 2013.

\section{Keywords}

Spectral quality; light source; wavelength; Salvia; Scarlet Sage.

\begin{abstract}
Three different qualities of light were analyzed on ornamental specie of economic and commercial importance: Salvia splendens or Scarlet Sage (variety Red \& White of PanAmerican Seeds(C), using three different sources of artificial light (blue-LED, purpleLED and fluorescent) to modify the growth environment of each one. The investigation covered analysis of days to flowering, height, diameter, number of buds and SPAD values. Existing studies on the effect of spectral quality on plant growth show that exist significant alterations on variables in photomorphogenic and plant development. For example: the dry weight of the plant, height, internode length, number of buds, flowering duration, number of stomata per plant and amount of reducing sugars and starches. However, in this study it was found that the spectral quality provided with supplementary light only has effect in days to flowering compared with control treatment, because reduce in one week the flowering days, but, has not effect between supplementary lighting treatments. Others variables studied (buds number, height, diameter and values SPAD) had not significant effect by quality spectral used ( $p>0.05)$. The study was made in the academic greenhouse ITESM, located in Monterrey, Nuevo Leon, Mexico, during the period of August to December 2013.
\end{abstract}




\section{Introducción}

La planta ornamental Salvia splendens pertenece a la categoría de beddings plants, las cuales son de gran importancia en la economía norteamericana y europea. En países como Estados Unidos, por ejemplo, esta categoría de ornamentales representa ventas de más de 1300 millones de dólares anuales (USDA, National Agricultural Statistics Service, 20 I3). Siendo una especie de alta importancia comercial, el estudio de efectos sobre su desarrollo vegetal adquiere importancia en términos de mejoramiento en la producción de las mismas. La variedad estudiada, vista red and white, tiene un denso crecimiento de sus espigas florales, follaje verde oscuro con crecimiento continuo, sin decoloración por la luz solar, buena tolerancia al calor, precoz y de costumbre (PanAmerican Seed, 2013).

La aplicación de diferentes fuentes de luz con distinta longitud de onda es una técnica para analizar algunas características en el desarrollo vegetal. De acuerdo con Gil (1995), Sinha (2004) y Hopkins (1995) las longitudes que producen sensación de luz se hallan entre los 390 y los 760 nm y los pigmentos fotosintéticos absorben sólo esta porción visible del espectro. Además, cualquier fuente de energía radiante que incluya longitudes de onda dentro del espectro visible inducirá fotosíntesis siempre y cuando tenga suficiente intensidad (Meyer \& Anderson, 1952). Las zonas de absorción máxima se encuentran en la región de onda corta y larga del espectro visible. En el centro se hallan los valores de longitud de onda que corresponden al color verde que, prácticamente, no se absorbe y de ahí la coloración verde que exhiben los vegetales (Gil, 1995).

Las luces tipo LED (Light Emitting Diode) son utilizadas actualmente porque ofrecen una especificidad en la longitud de onda y un calentamiento mínimo y han sido de gran utilidad en estudios previos con beddings plants (Heo, Lee, Chakrabarty, \& Paek, 2002; Heo, Lee, \& Paek, 2006). En el caso de la luz azul (LA), la longitud de onda emitida tiene un rango entre 425 y 490 nm (Hopkins, 1995) y la luz púrpura (LP), al ser una combinación de azul y rojo emite rangos de 425 a $490 \mathrm{~nm}$, correspondiente al color azul, y de 640 a 700 nm que corresponden al color rojo (Hopkins, 1995). Por otra parte, la luz fluorescente ha sido la fuente de luz artificial más común en las investigaciones con plantas superiores, sin embargo, este tipo de luz ofrece únicamente un amplio espectro de radiación para las plantas (Heo et al, 2002).

La clorofila, al ser el principal pigmento fotosintético en los vegetales, va a estar afectada por la calidad espectral percibida. De acuerdo con Gil (1995), Sinha (2004) y Hopkins ( 1995) la clorofila a posee máximos de absorción entre 430 y 440 nm y luego a 600 nm; por su parte la clorofila b entre los 450 y 460 nm y luego entre 640 y 650 nm. Por esta razón, se consideró realizar un análisis no destructivo del contenido clorofilico bajo los distintos tratamientos mediante el dispositivo SPAD-502. Dicho dispositivo fue desarrollado por la compañía japonesa Minolta Camera $@$ y da lecturas en unidades denominadas SPAD por sus siglas en inglés (Soil-Plant Analyses Development). Este dispositivo portátil, ligero, con auto-calibración y no destructivo registra mediciones de densidad óptica en dos distintas longitudes de onda, las cuales convierte en señales digitales y posteriormente en valores SPAD (Rodriguez \& Miller, 2000). Varios autores como Cerovic, Masdoumier, Ben Ghozlen, \& Latouche (20 I2); Rodriguez \& Miller (2000); Mielke, Schaffer, \& Li (20 I0); Ling, Huang, \& Jarvis (20 I I) y Uddling, Geland-Alfredsson, Piiki, \& Pleijel (2007) reportan

Cuadro I. Integral de luz diaria y temperatura promedio recibida de todos los tratamientos durante 36 días.

\begin{tabular}{|c|c|c|c|c|}
\hline Factor & LFI* & LP* & LA* & T* \\
\hline Luminosidad $\left(\mathrm{mol} \cdot \mathrm{m}^{-2} \cdot \mathrm{dia}^{-1}\right)$ & 19.84 & 20.77 & 19.87 & 22.49 \\
\hline Temperatura día $\left({ }^{\circ} \mathrm{C}\right)$ & 30.37 & 30.25 & 30.13 & 29.91 \\
\hline Temperatura noche $\left({ }^{\circ} \mathrm{C}\right)$ & 22.26 & 22.19 & 22.25 & 21.99 \\
\hline
\end{tabular}

*Los cálculos se hicieron basado en los datos de los datalogger Hobo tipo Pendant@ de OnsetTechnologies@ 
que efectivamente existe una relación entre el valor SPAD y el contenido de clorofila, tanto de clorofila a como clorofila b. Sin embargo, estos valores pueden ser afectados por el cultivar, estado de crecimiento de las plantas, factores edáficos y climáticos que puedan causar clorosis en las hojas, grosor de la hoja, suculencia foliar, masa foliar específica (Marenco, Antezana-Vera, \& Nascimento, 2009), hora del día en la que se hace la medición y el nivel hídrico de la planta (Martinez \& Guiamet, 2004).

El objetivo del estudio es analizar el efecto de diferentes calidades de luz sobre el desarrollo vegetal de las plantas mediante el estudio de cambios significativos entre los tiempos a floración, número de brotes, altura, diámetro promedio y valores SPAD.

\section{Materiales y métodos}

El presente estudio se desarrolló en el invernadero académico del Departamento de Agrobiotecnología del Tecnológico de Monterrey, México, localizado a

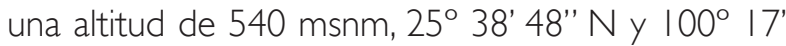
I" O Se evaluó el efecto de la calidad espectral de distintas fuentes lumínicas durante el desarrollo de la planta en estudio. Se conformaron tres ambientes de desarrollo mediante el uso de mini-túneles donde la fuente de emisión de luz artificial modificó el ambiente de crecimiento y un ambiente sin túnel como testigo. Los mini-túneles fueron construidos con tubería PVC hidráulica de 3/4" cedula 40, conformando una estructura de tres arcos de $2 \times$ $2.5 \times 1.6 \mathrm{~m}$ (L;A;A). Las fuentes lumínicas de 125 $W$ de luz fluorescente $(\mathrm{LFI})$ de Hydrofarm ${ }^{\circledR}$ para el ambiente I, de $32 \mathrm{~W}$ de luz azul (LA) de LedKesill para el ambiente 2 y de $32 \mathrm{~W}$ de luz púrpura (LP) de LedKesill $@$ para el ambiente 3; se instalaron sobre un riel de desplazamiento continuo montado en los mini-túneles y dispuesto a $80 \mathrm{~cm}$ sobre los materiales en estudio. Se utilizó una especie de paisajismo ornamental denominada Salvia ( variedad específica utilizada fue Vista Red \& White de Panamerican Seeds@.Se establecieron un total de 40 plantas distribuidas en lotes de 10 plantas cada una con un ambiente distinto (luz fluorescenteLFI, luz azul-LA, luz púrpura-LP y testigo-T). Se utilizó una mezcla de sustratos compuestos de dos partes de perlita por una parte de Peat Moss ${ }^{\circledR}$. En la solución nutritiva se utilizó 144 ppm de Nitrógeno (N), 120 ppm de Fosforo (K), 100 ppm de Potasio (P),
50 ppm de Azufre (S) y 80 ppm de Magnesio (Mg). Se utilizó el sistema Xilema@ para el riego automatizado, proporcionando $70 \%$ de solución nutritiva y $30 \%$ agua, balanceando además el pH en forma constante a un nivel de 6,I.

El sensor utilizado para el monitoreo de las condiciones ambiente fue el data loggers Hobo tipo Pendant ${ }^{\circledR}$ de Onset Technologies@), el cual permitió el monitoreo oportuno en in situ del ambiente físico existente durante el desarrollo de los cultivos (temperatura y luminosidad). Se utilizó el sensor SPAD-502Plus $®$ de Konica Minolta@ para registrar los valores SPAD medidos de forma aleatoria en las hojas de las plantas.

La recopilación de información se realizó a lo largo de 5 semanas en el caso de las lecturas SPAD. Los demás datos fueron recolectados a los 36 días posteriores al trasplante. Se realizó análisis de varianza (ANOVA) y agrupación por el método de Tukey con la finalidad de determinar diferencias significativas entre los distintos tratamientos.

\section{Resultados y discusión}

Dado que las diferentes fuentes de luz varían en calidad e intensidad y que cada cultivo tiene un rango óptimo en el que se maximiza la fotosíntesis (Torres \& Lopez, 2004), se calculó la luz diaria integrada, DLI por sus siglas en inglés, para cada tratamiento (luz fluorescente-LFl, luz púrpura-LP, luz azul-LA y tratamiento testigo- $\mathrm{T}$ ).

\section{Tiempo a floración}

La luz, a través de la fotosíntesis, influye en el crecimiento de las plantas así como en otros procesos de desarrollo vegetal como la germinación y la floración (Runkle \& Blanchard, 2013). En este estudio, se observó que LFI y LP lograron una floración más temprana respecto a LA y T. Sin embargo, una semana más tarde el tratamiento LA alcanzó a los otros tratamientos con fuentes lumínicas artificiales. El tratamiento $T$ mantuvo su nivel de floración en esa semana. La Salvia splendens al ser una especie que presenta una respuesta fotoperiódica facultativa (Runkle \& Blanchard, 2013) presentó una floración más rápida en los tratamientos que involucran luz artificial que el tratamiento testigo. No obstante, entre los tres tratamientos con luz artificial hubo una mínima diferencia en la floración a los 22 días 
(figura I) pero, a los 30 días ya se encontraban todos casi al mismo nivel de floración.

Número de brotes, altura y diámetro promedio

De acuerdo con el análisis estadístico realizado, no hay diferencia significativa entre el número de brotes, altura y diámetro de la Salvia splendens bajo los cuatro distintos tratamientos aplicados. Algunas investigaciones (Heo et al, 2002; Heo et al, 2006) han demostrado que existen efectos significativos de la calidad espectral sobre el crecimiento y la fotomorfogénesis en variables como: peso seco, altura, longitud entrenudos, número de brotes, duración del periodo de floración, número de estomas por planta y cantidad de almidones y azúcares reductores; en cultivares como: Salvia, Marigold y Ageratum. Sin embargo, con la metodología y dispositivos usados en este experimento, no efectos significativos.

\section{Valores SPAD}

De acuerdo con el análisis estadístico, no existe diferencia significativa en ninguno de los diferentes tratamientos en cuanto a los valores SPAD obtenidos semanalmente con el SPAD-502. Estudios anteriores (Ling et al, 20 I I; Cerovic et al, 20 I2; Uddling et al, 2007) han establecido relaciones lineales y no lineales entre el valor SPAD y el contenido de clorofila. Por lo tanto, la calidad espectral, en este caso, no influyó en la cantidad de clorofila en los distintos tratamientos y por ende no afectó la capacidad de absorción de luz para el proceso de fotosíntesis.

\section{Conclusión}

Los tratamientos con luz artificial utilizados (LFI, LA, LP) aceleran los días a floración de 22 a 30 días en comparación con el tratamiento testigo. Sin embargo, entre los tratamientos LFI, LA y LP no hay nin-

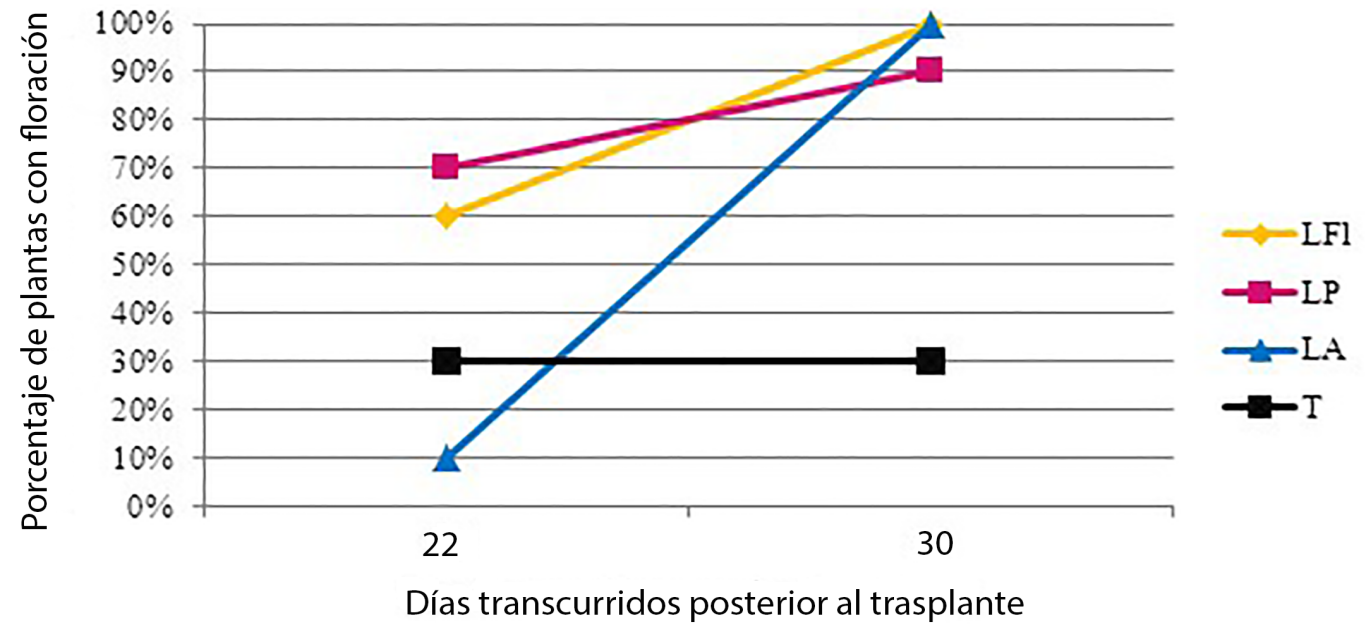

Figura I. Porcentaje de plantas en floración a los 22 y 30 días.

Cuadro 2. Resultados de número de brotes, altura y diámetro en los distintos tratamientos (luz fluorescente-FLI, luz azul-LA, luz púrpura-LP y testigo-T).

\begin{tabular}{|c|c|c|c|c|}
\hline Variable & LFI & LP & LA & T \\
\hline Brotes $(\mathrm{u}) *$ & $4.30 \pm 0.95(\mathrm{a})$ & $3.60 \pm 0.70(\mathrm{a})$ & $4.40 \pm 1.17$ (a) & $3.90 \pm 1.37(\mathrm{a})$ \\
\hline Altura $(\mathrm{cm})^{*}$ & $32.90 \pm 3.32(\mathrm{a})$ & $34.40 \pm 1.78(\mathrm{a})$ & $34.50 \pm 1.51$ (a) & $35.10 \pm 2.96(\mathrm{a})$ \\
\hline Diámetro $(\mathrm{cm}) *$ & $28.25 \pm 3.06(\mathrm{a})$ & $28.25 \pm 3.26(\mathrm{a})$ & $28.95 \pm 3.26(\mathrm{a})$ & $27.75 \pm 5.28(\mathrm{a})$ \\
\hline
\end{tabular}

Los valores que comparten una letra no son significativamente diferentes. *Valor de $p>0.05$ 
Cuadro 3. Valores SPAD a lo largo de 5 semanas en los distintos tratamientos (luz fluorescente-FLI, luz azul-LA, luz púrpura-LP y testigo-T)

\begin{tabular}{|c|c|c|c|c|}
\hline Semana & LFI & LP & LA & $T$ \\
\hline Semana I* & $44.59 \pm 4.27(\mathrm{a})$ & $44.35 \pm 5.36(\mathrm{a})$ & $45.33 \pm 3.16(\mathrm{a})$ & $42.85 \pm 4.5$ I (a) \\
\hline Semana 2* & $43.98 \pm 5.20(\mathrm{a})$ & $43.70 \pm 2.99(\mathrm{a})$ & $46.09 \pm 4.52(\mathrm{a})$ & $40.96 \pm 6.44(\mathrm{a})$ \\
\hline Semana 3* & $44.97 \pm 5.76(\mathrm{a})$ & $46.62 \pm 2.52(\mathrm{a})$ & $45.03 \pm 4.88(\mathrm{a})$ & $40.59 \pm 5.44(\mathrm{a})$ \\
\hline Semana 4* & $46.80 \pm 5.21(\mathrm{a})$ & $49.12 \pm 2.98(\mathrm{a})$ & $46.64 \pm 1.86(\mathrm{a})$ & $46.92 \pm 2.88(\mathrm{a})$ \\
\hline Semana 5* & $49.36 \pm 5.48(\mathrm{a})$ & $49.48 \pm 4.85(\mathrm{a})$ & $45.54 \pm 8.01(\mathrm{a})$ & $50.29 \pm 3.97(\mathrm{a})$ \\
\hline
\end{tabular}

Los valores que comparten una letra no son significativamente diferentes. *Valor de $p>0.05$

guna diferencia. Por otra parte, la calidad espectral utilizada no tiene ningún efecto sobre el número de brotes, altura, diámetro y valores SPAD en la Salvia splendens var.Vista Red and White.

\section{Bibliografía}

Cerovic, Z., Masdoumier, G., Ben Ghozlen, N., \& Latouche, G. (20।2). A new optical leaf-clip meter for simultaneous nondestructive assessment of leaf chlorophyll and epidermal flavonoids. Physiologia Plantarum, 146, 251-260.

Gil, F. (1995). Elementos de Fisiología Vegetal. Madrid: MundiPrensa.

Heo, J.W., Lee, C.W., \& Paek, K.Y. (2006). Influence of mixed LED radiation on the growth of annual plants. Journal of plant biology, 49(4), 286-290.

Heo, J., Lee, C., Chakrabarty, D., \& Paek, K. (2002). Growth responses of marigold and salvia bedding plants as affected by monochromic or mixture radiation provided by a LightEmitting Diode (LED). Plant Growth Regulation, 38, 225-230.

Hopkins, W. (1995). Introduction to plant physiology. New York: John Wiley \& Sons.

Ling, Q., Huang, W., \& Jarvis, P. (20 I I ). Use of a SPAD-502 meter to measure leaf chlorophyll concentration in Arabidoptis thailiana. Photosynth Res, 209-2I4.

Marenco, R., Antezana-Vera, S., \& Nascimento, H. (2009). Relationship between specific leaf area, leaf thickness, leaf water content and SPAD-502 readings in six Amazonian tree species. PHOTOSYNTHETICA, 47(2), 184- 190.

Martinez, D., \& Guiamet, J. (2004). Distortion of the SPAD 502 chlorophyll meter readings by changes in irradiance and leaf water status. Agronomie, 24, 4I-46.
Meyer, B., \& Anderson, D. (1952). Plant physiology. New York: D. Van Nostrand Company.

Mielke, M., Schaffer, B., \& Li, C. (20 I 0). Use of a SPAD meter to estimate chlorophyll content in Eugenia uniflora L. leaves as affected by contrasting light environments and soil flooding. PHOTOSYNTHETICA, 48(3), 332-338.

PanAmerican Seed. (2013). PanAmerican Seed. Recuperado el 2 de II de 2013, de Vista red and white Salvia: http://www. panamseed.com/plant_info.aspx?phid $=05490$ | 62 I 004575 \& dispmode $=$

Rodriguez, I., \& Miller, G. (2000). Using a Chlorophyll Meter to Determine the Chlorophyll Concentration, Nitrogen Concentration, and Visual Quality of Augustinegrass. HortScience, 33(5), 75 I-754.

Runkle, E., \& Blanchard, M. (I7 de Abril de 2013). Greenhouse Energy Conservation Strategies: Lighting. Recuperado el 10 de Noviembre de 2013, de Use of lighting to accelerate crop timing: http://www.hrt.msu.edu/energy/Notebook/pdf/Secl/ Runkle\%20and\%20Blanchard,\%20Use_of_Lighting.pdf

Sinha, R. (2004). Modern Plant Physiology. India: Alpha Science International Ltd.

Torres, A., \& Lopez, R. (2004). Floriculture Purdue University. Recuperado el I 4 de II de 2013, de Medición de luz diaria integrada en invernaderos: http://www.extension.purdue. edu/extmedia/HO/HO-238-SW.pdf

Uddling, J., Geland-Alfredsson, J., Piiki, K., \& Pleijel, H. (2007). Evaluating the relationship between leaf chlorophyll concentration and SPAD-502 chlorophyll meter readings. Photosynth Res, 37-46.

USDA, National Agricultural Statistics Service. (Abril de 2013). Floriculture crops. Recuperado el 2 de II de 2013, de Summary 2012: http://usda0l.library.cornell.edu/usda/current/FlorCrop/FlorCrop-04-25-2013.pdf 